\title{
Robotic Locomotion Systems, Unconventional
}

\section{An Introduction to Robotic Locomotion \\ II. Why Develop Unconventional Robotic Locomotion Systems? \\ III. Terrestial/Overland Robotic Locomotion \\ IV. Underwater Robotic Locomotion \\ V. Aerial and Aerospace Robotic Locomotion \\ VI. Hints of the Future}

\section{GLOSSARY}

Biomimetic Inspired by or derived from observations of biologically based mechanisms occurring in nature.

Gait A cyclic motion in the internal shape of a robot that leads to net changes in its position and velocity.

Hybrid locomotion The use of multiple modalities to generate locomotion, e.g., wheels and legs, swimming and flying, etc.

Pose The position and orientation of the robot, generally taken with respect to some inertial frame.

Robotic locomotion The process by which a robotic system moves through its environment.

Shape The components of the internal geometric configuration of a robot that are used to generate mobility.

Unconventional robotic locomotion Robotic locomotion systems that do not rely on human-based locomotion modalities such as wheels or legs.

\author{
James P. Ostrowski \\ University of Pennsylvania
}

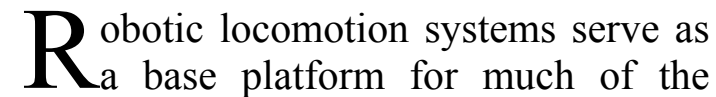
field of robotics. Although the general public notion of a robot usually conjures images of either walking bipeds or wheeled mobile robots, the types of systems developed in robotics research extend far beyond these two classes of robots. Starting from a very general definition of gait, one finds that it is quite natural to define a wide variety of machines and devices as qualifying as robotic locomotion systems, including swimming robots, satellites with rotors, and modular metamorphic robots. 


\section{An Introduction to Robotic Locomotion}

"The journey of a thousand li starts

with a single step."

— Lao Tzu, Tao-te Chung

From the early inventions of mobile machinery, the fundamental desire to include mobility into our mechanical systems has often played a central role in design. With the growth of robotics as a research field of its own in the 1950's and early 1960 's, the focus shifted towards enabling the machines to move under their own control, or at least under the remotely supervised control of a human user.

While the field has grown and changed in many ways since its birth, the goal of incorporating mobility into the robotic systems has remained fundamental. We will use a very broad definition of robotic locomotion, requiring only that the system be able to actively alter or regulate its position and orientation in space. This is perhaps the intuitive definition one would naturally arrive at to describe a system which locomotes, or moves through its environment. It immediately distinguishes robotic locomotion systems from the class of fixed-base robots that one associates with assembly lines and industrial manufacturing. Such robots primarily have a robotic arm working from a rigidly fixed base platform, and are used to manipulate objects, weld or insert parts, inspect hard to reach places, or perform other tasks, often in association with some type of assembly line operation.

What our definition of robotic locomotion does include, however, is many of the obvious types of robotic systems that are commonly seen in science fiction - the kind conjured up in movies like Star Wars, such as legged robots (think "C3PO") and wheeled robots (think "R2D2"). But it also refers to a diverse spectrum of other types of robots. Some of these are familiar to us, like snakes, inchworms, frogs, birds, and insects, because they are derived from biological inspirations. Some, on the other hand, may at first glance not seem to be locomotion systems, since they do not "move" their position at all, but only reorient. Consider, for example, a satellite in space that only needs to orient itself-we will include this type of system in our broad definition of locomotion. And beyond this, there will be some systems whose modes of locomotion have never been observed in nature, but which are spawned from the creative imaginations of modern designers and roboticists.

In the remainder of this section, we give a fairly technical description of the fundamentals of robotic locomotion. The reader only curious in learning about specific details of existing unconventional robotic locomotion systems should feel free to skip ahead to Section II. The important terms used in the text are highlighted in this section, and also are briefly defined in the Glossary above.

To start with a more formal definition of robotic locomotion, let us pick an inertial reference frame in space and attach a second reference frame to the body of the robot. Then locomotion of the robot requires that it be capable of generating changes in the relative displacement between the two reference frames and/or changes in the orientation between the two frames. For example, the motion of a satellite in space could involve full spatial positioning and orientation maneuvers, implying six degrees of freedom. Most locomotion 
systems, however, don't involve this many degrees of freedom. Some common examples are wheeled planar mobile robots, whose motion is restricted to a single plane of motion, and examples of satellites in which only the reorientation is considered.

This simple description has led to a general framework that can be used to describe a wide variety of locomotion systems. Developed by Ostrowski and Burdick, the underlying principle is to decompose robotic locomotion into two primary components, namely the pose (position and orientation) of the robot, and the shape, or internal configuration of the robot. The shape of the system is often taken to be simply the remainder of the configuration space of the robot once the pose has been factored out. Thus, it can be the rotation and steering angle of the wheels in a car-like robot; the joint angles of the legs on a walking robot; the internal bending or curvature of a snakeor eel-like robot; the control surfaces such as the rudder, elevator, and ailerons for an airplane; or reorientation devices such as momentum wheels found in a satellite.

Given these two definitions, the theoretical framework for locomotion comes down to describing how the changes in the internal shape lead to changes in the pose of the robot. For some systems, this can be described kinematically as a direct relationship between the motion of the internal shape and the changes in position and orientation. This is the case for wheeled robots. Assuming there is no slippage between the wheels and the ground, the wheel velocities directly imply the speed at which the body moves. Models of paramecia swimming in highly viscous fluids also can be expressed in a kinematic form, where the shape can be thought of as the closed curve representing the perimeter of the paramecium's body. Deformations of the body (actually, fluctuations of hairlike cilia around the body) lead directly to changes in its position.

In other cases, the changes of shape lead indirectly to motion, through the dynamics of the system. Very often this is through a dynamic interaction with the environment. For example, in running or hopping, there are forces that are generated as the robot pushes off the ground. Likewise, the motion of an eel uses internal shape changes - in this case a traveling wave sent down the length of the body - to generate propulsive forces. These forces can be modeled by drag forces on each segment of the body, as well as by forces generated as vortices that are shed into the wake of the moving robot. Dynamics are also equally important in flying, where cyclic changes in the shape, through flapping of wings, for example, can lead to net forces that enable flight. Even for systems that use lifting surfaces or buoyancy effects, such as airplanes and blimps, respectively, the role of shape is an important one. For airplanes, the shape determines the effect of the lifting surfaces, as the control surfaces alter the wind flow and thus the forces generated on the airplane. For the blimp, directional thrusters are often used, and the direction they point can be thought of as a shape input. The thruster action itself, though, provides a force applied directly to the pose, and so is generally not considered to be a shape input. Instead, it is treated as a separate control input that can directly change the pose of the system through its dynamics.

Motivated by biological observations of horses and humans, roboticists have also asked the question of what types of 
inputs could be considered generic, or fundamental, to locomotion systems.

For biologists, the question is made somewhat easier, since one only needs to observe and classify the motions found in nature. These observations for legged mammals have led to the classification of gaits, which are essentially different patterns of motion of the shape that lead to locomotion. In quadrupeds, such as horses, these gaits are generally described by the phasing between the legs. For example, a horse will generally run in one of several gaits, including the trot, gallop, or pace, with each gait described by the relative phasing with which the feet touch the ground. There is even the very unusual gait used by gazelles called the pronk, in which all four legs strike the ground simultaneously, to generate a hopping motion. Similar descriptions of gaits have been used for humans (consider the walk, skip, or hop), and for creatures with more than four legs. Hexapods, for example, often use a tripod gait, whereby two sets of three legs (each forming a tripod of support) move in alternating patterns.

The notion of gait is a powerful one for robotic locomotion, since it generally describes classes of inputs that can be used to generate desired motions. As such, we extend the notion of gait to general locomotion systems by describing it as a cyclic pattern of shape changes that lead to motion (changes in the pose) of the system. With this interpretation, we see that many systems naturally have gaits that are used in their motion. Examples range from the serpentine motions of a snake or eel to the cilial deformations of a paramecium moving through a viscous fluid to the use of momentum wheels in satellites to control orientation. We also can then speak of classes of gaits for individual systems that generate qualitatively distinct motions. Consider a wheeled mobile robot that is able to turn about its center point (see the Urbie in Figure 4). Rolling both wheels forward at the same speed leads to forward motion. Moving the wheels in opposite directions at equal speeds provides a pure rotation. Finally, one can couple the two motions, as is done in parallel parking, to generate a sideways, lateral motion. These are three "gaits" that exist for the wheeled mobile robot. Similar descriptions exist for a variety of other systems. For example, a snake robot can inchworm forward (shown in Figure 3 and Figure 10), use a serpentine gait (shown in Figure 2), or use more exotic, rolling type gaits such as sidewinding. Similarly eel robots (shown in Figure 10) can thrust forward, as well as generate rotational and lateral motions using different types of gaits. One can also think of locomotion systems that don't have any translational motion at all, but only rotate. Good examples of this are objects in space, such as satellites, that have zero linear and angular momentum. Even though the angular momentum is constrained to be zero, one can consider locomotion of the system that involves reorientation through the use of shape changes, or gaits. To visualize this, consider an astronaut floating in space who wants to reorient to face in the opposite direction. By moving his arms in a circular motion, similar to stirring a boiling kettle, the astronaut will rotate around in the opposite direction. This gait, which involves a periodic motion of the shape (in this case the arms), leads to a net change in pose through reorientation. Satellites perform similar motions through the use of momentum wheels. Turning a wheel attached to the satellite 
generates a reaction moment that rotates the satellite in the opposite direction. This simple gait gives reorientation. Furthermore, mounting two wheels on orthogonal axes of the satellite allows one to generate motions about the third, unactuated axis of the satellite. This is done by properly coupling the inputs of the first two wheels, giving rise to a "gait" for satellite reorientation!

We also mention a final interesting point that is common to most, if not all, locomotion systems. Regardless of how the inputs and shape effects enter into the system, the equations of motion can be simplified by using the decomposition into shape and pose. The important characteristic to recognize is an invariance of the motion with respect to the pose - in other words, it doesn't matter where the system starts out in reference to the inertial frame; only the relative motions of the body are affected by the inputs and the forces applied to it. This invariance leads to a significant reduction in the equations of motion that are required to model the system, and often simplifies the control and navigation of such systems.

\section{Why Develop Unconventional Robotic Locomotion Systems?}

"When man wanted to make a machine that would walk, he created the wheel, which does not resemble a leg."

- Guillaume Apollimaine, Les Mamelles de Tiresias (1918)

For the purpose of this discussion, the distinction between "conventional" and "unconventional" robotic locomotion will be left relatively informal. In the current context, we will, with notable exceptions, take the viewpoint that "conventional" implies systems in which the robot follows either of the two primary modes that humans use to move: walking, through the use of legs, and rolling, such as is found in bicycles and automotives.

The motivations for studying what we have termed unconventional locomotion systems are wide ranging, but most often they have to do with efficiency, agility, robustness, adaptability, and suitability (of unconventional locomotion over other types of "conventional" systems). For example, the efficiency achieved in swimming by biomimetic locomotion systems has the potential to far exceed our current engineering technologies. We will also discuss below several systems that are not biomimetic systems, but which have the potential to exhibit efficiencies far greater than traditional, conventional locomotion. These primarily use the notion of controlled buoyancy to generate locomotion forces requiring very low power. Efficiency plays a critical role in autonomous robotic devices, since battery life continues to be a dominant factor in restrictions on the duration of the remote operation of mobile robots.

Biomimetic and other types of robotic systems also can yield increased agility, as they are often designed with this as a primary goal. For example, swimming systems based on the pike or eel have the potential ability to make rapid turns and darting maneuvers that cannot be achieved through traditional propellers and conventional steering.

Issues of robustness and adaptability can also be quite important in robotic locomotion. The ability to move through environments that are not just smooth, even planar surfaces is a requirement for many robots working in natural terrains or environments. For 
this reason, conventional wheeled robots are ill-suited to navigate the surface of Mars or even the front steps at the local science museum. Legged systems are commonly studied to get around this problem, though with limited success to date. However, there are several systems out there that address just these issues of extending the capability of robots to work robustly in widely varying environments, while attempting to maintain the efficiency and simplicity found in conventional robotic systems.

On the other hand, there are many environments in which legged or wheeled systems are obviously inappropriate. This is often the case in underwater activities, but is certainly true universally when one looks at aerial robotic systems, such as planes and helicopters, and aerospace vehicles, such as satellites and deep space probes.

Lastly, there are numerous robotic systems that can be labeled as curiosities, but which fundamentally explore deeper issues in dynamics, modeling, and control. Examples of these are also discussed below, with some hints as to the context within which they were developed.

Our goal, then, is to explore a variety of robotic locomotion systems that utilize a very wide range of mechanisms and modalities for generating motion. Such systems are divided roughly according to the environments in which they operate; namely, terrestrial, underwater, and aerial/aerospace. The descriptions are necessarily neither comprehensive, nor complete, but are intended to provide an insight into a breed of modern robotic systems that are less often seen in the public media spotlight.

\section{Terrestial/Overland Robotic Locomotion}

"The distance is nothing; it is only the first step that is difficult."

- Madame du Deffaud, 1763 (on walking with one's head cut off).

Most conventional robotic locomotion systems move over dry land, and many are tailored to working in well-structured environments such as offices or warehouses. In such cases, the ground is generally flat and smooth, and a wheeled robot more than suffices.

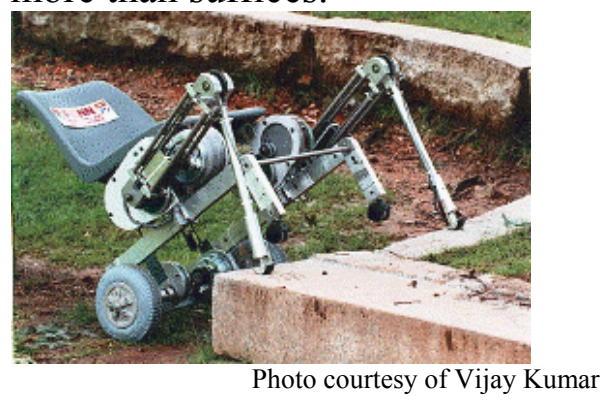

Figure 1: Walking wheelchair

A class of robots that is most closely associated to wheeled or legged systems is characterized by hybrid robotic locomotion systems. Most commonly, this refers to some type of wheel-legged hybrid, such as the walking wheelchair developed by Krovi and Kumar, shown in Figure 1.

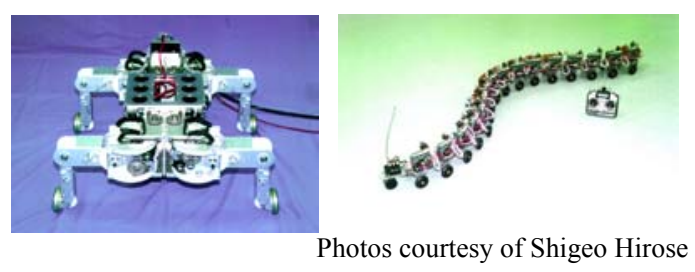

Figure 2: Hirose's wheel-legged hybrid

Other similar systems have been developed, for example, by S. Hirose at the Tokyo Institute of Technology. One such robot uses wheels at the end of a set of legs, as shown in Figure 2. When it is 
necessary to climb over obstacles, this robot can walk as a quadruped robot, with the wheels turned face down to the ground (the wheel axis points into the ground). However, it can also turn the wheels upright and use them to roll over even terrain. This combines the efficiency of wheeled locomotion with the terrain adaptability of legged locomotion for climbing over obstacles . Also shown in Figure 2 is a snake robot developed by Hirose, called the ACM. This robot was the first fully developed unconventional mobile robot to move overland. A significant body of work in the area of snake-like robots has also been done by Burdick, Chirikjian, Choset, and others. These robots, often referred to as hyper-redundant robots for their many extra degrees of freedom, are capable of serpentine, inchworm (shown in Figure 3), and side-winding motions, to name a few.

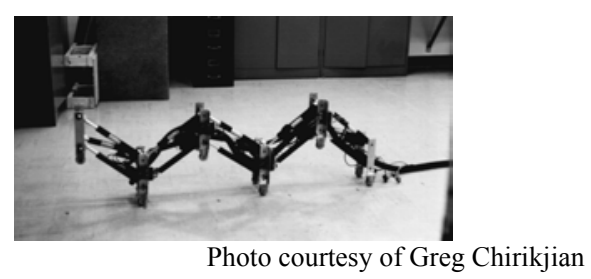

Figure 3: Inchworm gait of snake-like robot

A slightly different take on the concept of hybrid locomotion systems has also been developed by ISRobotics, founded by Brooks of MIT, and known primarily for its legged systems Genghis and Attila. The URBAN Build, or "Urbie," is shown in Figure 4. It is a track-legged hybrid system that uses the tracks for locomotion over very rugged terrain, but can employ the front legs (called "flippers") to climb up and over objects such as large curbs or stairs. The flippers also enable the Urbie to stand upright, like a squirrel standing on its hind legs, in order to get a better vantage point. The developers also are building the Urbie to "fly," similar to the flight of many land-based mammals, in a direction strictly bound by gravity - the robot is tossed by an operator out the window or over a fence, and is designed to survive the fall.

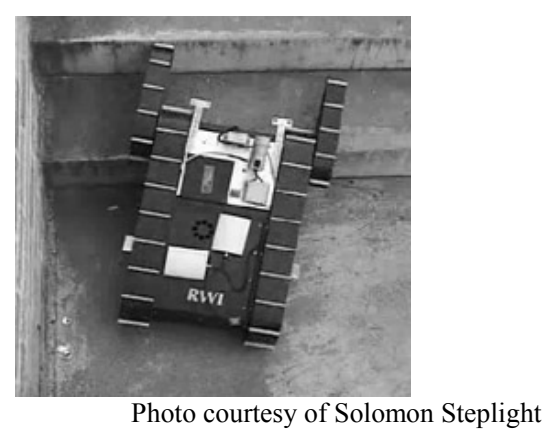

Figure 4: ISRobotics' Urban Build

The fact that the Urban robot was developed by a private company also highlights an interesting point - the place of locomotion within the field of robotics is currently in a state of rapid change, as commercial companies, including larger corporations such as Sony, Honda, and Mitsubishi, are developing mobile robotic systems. The resources and talents available from these companies may lead to the creation of a higher quality standard of robotic systems than can traditionally be delivered through an academic or small company mechanism.

For example, Honda's Humanoid robot, shown in Figure 5, demonstrates new levels of flexibility and robustness in bipedal walking, while Sony's AIBO dog-like entertainment robot (also shown in Figure 5) is the first really to target the general consumer audience, having sold an unheard of 3000 robots in under 30 minutes. Although these robots fall into the class of robots that we have characterized as "conventional" robotic 
locomotion systems, we note that Sony has developed the AIBO robot as a modular platform. Thus, it can potentially have wheels or other devices mounted on the body instead of legs, to form a hybrid system. Mitsubishi Heavy Industries has also recently entered the field of robotic locomotion systems, having developed swimming robots that are based on extinct species of fish. It is not clear what the commercial potential for such systems will be, though there is a large interest currently in underwater robotic exploration.

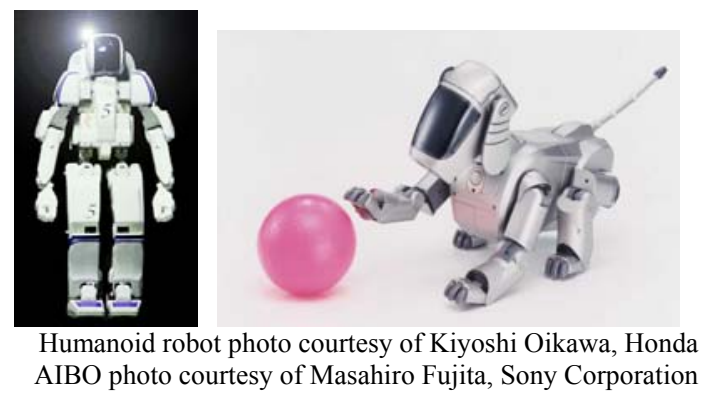

Figure 5: Honda's Humanoid Robot and Sony's AIBO Entertainment Robot

Two even odder modes of locomotion have been demonstrated using mechanisms that rely on transfer of angular momentum to linear momentum. In both cases, the robots utilize momentum, along with a frictional contact with the ground, to generate displacements and velocities.

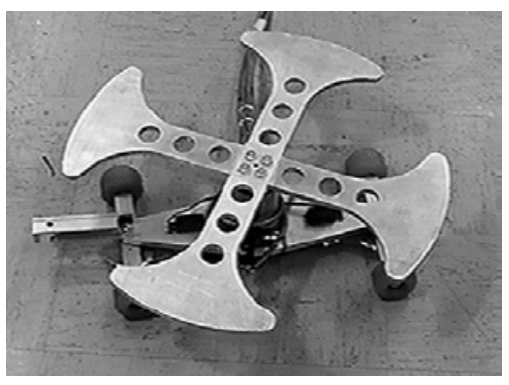

Figure 6: Snakeboard robot
The snakeboard robot, shown in Figure 6 , evolved from a commercial variant of the popular skateboard. Developed in South Africa to allow skiing aficionados the opportunity to practice their sport even in the middle of summer, the commercial Snakeboard requires a careful coupling of twisting the torso (or in the case of the robot, the spinning of a momentum wheel) with turning of the wheel trucks. Doing so allows the rider, and hence the robot, to generate a forward motion reminiscent of the serpentine gait of a snake. Unlike snake robots, the snakeboard can then coast and also make very sharp turns, limited only by the turning radius of the two sets of wheels. A similar system was studied by Krishnaprasad and Tsakiris, based on a children's toy called the Tennessee Roller Racer. These systems provide important examples in the robotics literature, as they were the first locomotion systems that clearly required a careful consideration of the dynamics, including accelerations and momentum transfer, as they affected the motion. The snakeboard also was shown to have a variety of gaits, or cyclic input patterns, that could be used. These gaits relied on coupling cyclic motions of the shape inputs at different, integrally related frequencies. Each gait resulted in a distinct motion, ranging from forward motion to parallel parking to rotating in place.

The sphericle, shown in Figure 7, on the other hand, utilizes a more direct momentum transfer. This system was originally studied by Bicchi, and also has been investigated as the "gyrover" by researchers at Carnegie Mellon Univeristy. The locomotion generated by such a device is akin to that used in an outdoor fair event popular among college students. In such an event, 
students are inserted in a large sphere and allowed to "walk" the sphere along the ground. The sphericle uses a similar mechanism to allow it to move along the ground by transferring the internal motions into external rolling.

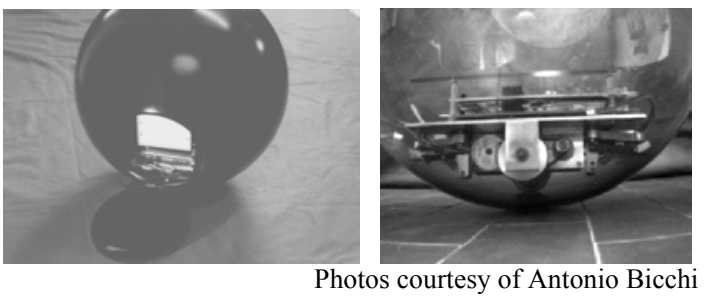

Figure 7: The Sphericle

If the sphericle is fully actuated with momentum wheels (in this case, it requires three wheels mounted orthogonally to each other), it can easily move in any direction. However, in the case that there are only two actuators present, a gait (what Bicchi refers to as a control quantum) can be found that generates motions about the third axis. That this can happen-coupling of a pair of inputs to generate motion in a third, independent direction - is fundamental to many classes of locomotion systems, including conventional systems involving wheels and legs. In fact, it is in many ways the reason why gaits are important as units of control for robotic systems-it is exactly the coupling of shape inputs that leads to changes in motion. In the case of the sphericle, it is the geometry (curvature) of the sphere that leads to this coupling. We will also see that a similar coupling occurs for reorientation of satellites in space.

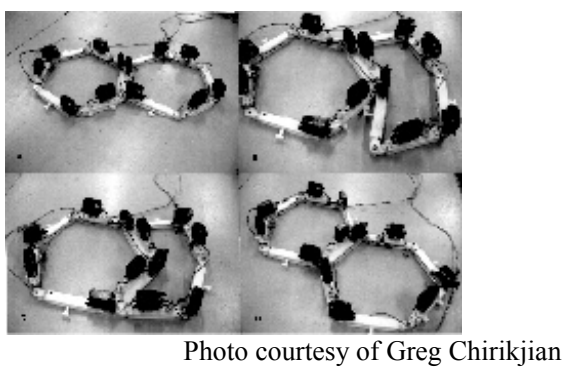

Figure 8: Robot metamorphosis

Lastly, we mention a class of robotic systems that are capable of locomoting over land-based terrain, but could provide a platform for moving through a variety of environments. These are what are known as metamorphic, or selfreconfigurable, robots. An example of such a robot developed by Chirikjian is shown during a reconfiguration stage in Figure 8. Studied from various perspectives by several researchers, including Yim, Chirikjian, Rus, and Murata, these robots are composed of many distinct, generally identical, subunits, called modules.
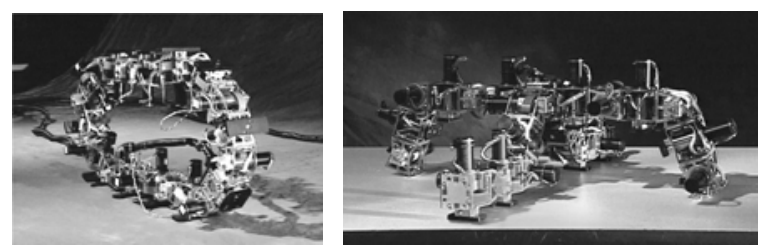

Photo courtesy of Mark Yim, Xerox Palo Alto Research Center

Figure 9: Modular robot in rolling and spider configurations

Although they are generally studied in relation to internal selfreconfiguration, with the goal of assuming any desired shape, locomotion is certainly achievable by moving modules along the body of the robot.

Taking this goal further, Yim and others have enumerated several classes of modular configurations capable of locomotion, along with their associated gait patterns. These robots are capable 
of forming different types of locomotion systems from the same set of basic molecules. Shown in Figure 9 are two configurations, one a circular, or loop, shape that locomotes using a rolling motion similar to a tank track, and the other a spider configuration that uses legged walking gaits akin to a spider.

\section{Underwater Robotic Locomotion}

"The reason why I love the sea, I cannot explain,"

- Jacques Cousteau

The ability to traverse a variety of landbased terrains is only a part of the locomotion picture - there are many more areas in which robotic systems can be useful in exploration, surveillance, and retrieval. The draw of the sea is one felt by many researchers. It is thus no surprise that the exploration of underwater environments is an area that has seen a great deal of activity lately. This is motivated by a variety of interests, including gaining a better understanding of the role played by the oceans in impacting our environment, weather patterns, and ecosystem; reconnaissance missions to sunken treasure and artifacts; and the need for search, rescue, and retrieval of items lost at sea, as has occurred all too frequently recently as a result of airplane crashes. Some of the "conventional" robotic systems that are used rely on propellers to provide the necessary forces to generate controlled motion through the water. There are many examples of these, though perhaps the best well known is the JASON robot developed at the Woods Hole Oceanographic Institute.

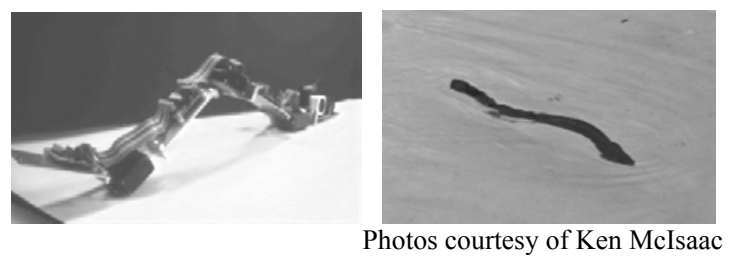

Figure 10: Eel robot shown inchworming (without skin) and swimming

Similar to land-based locomotion, where hybrid robots were used to enable motions over more than just flat, even ground, a new generation of robotic devices has been tailored towards transitioning from land to water. These are exemplified by the lobster robot, developed by Ayers at Northeastern University, and the snake- or eel-like robot developed by McIsaac and Ostrowski, shown in Figure 10. The lobster robot primarily relies on walking for its mobility, and so is restricted to motion along the sea floor. The eel robot utilizes a serpentine gait, found in land- and water-based snakes. This allows it to slither along soft terrain, such as a beach, and then move into water directly. It is also capable of inchworming across other types of terrain or over obstacles, as shown in Figure 10.

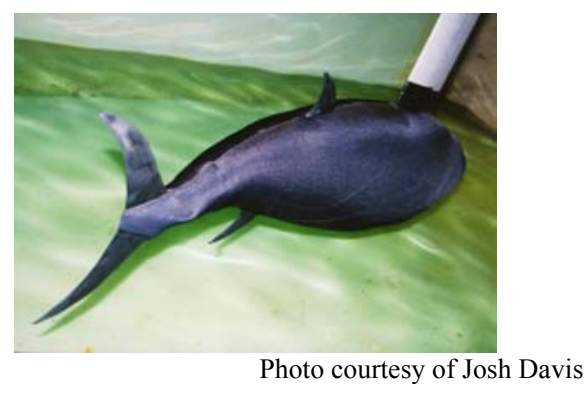

Figure 11: RoboPike

There are many other types of underwater robotic systems that provide a diverse array of abilities. Like the lobster and eel, the design and control of 
these robots are often motivated by principles derived from biological observation, and so are referred to as biomimetic systems. One of the first examples of such a device was the RoboTuna, which was later followed by the RoboPike (shown in Figure 11), based on full-scale models of tuna and pike. The RoboTuna was purely a towed device, and so did not actually "swim," though it provided ample data suggesting the increased efficiency of such a biomimetic propulsion source. The RoboPike was later developed to explore the extent to which fish-like propulsion schemes can increase the agility of the robotic device, since pikes are known for their sharp turning angles and quick startup speeds.

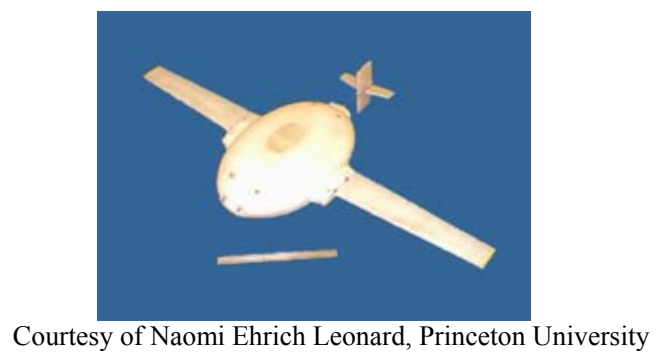

Figure 12: Buoyancy controlled underwater device

A final device that deserves some attention is not based on biological observations, but does yield a highly efficient mechanism for underwater locomotion. This robot, developed by Leonard at Princeton University, uses an interesting mechanism for propulsion, that only requires that the buoyancy force on the robot be changed. By increasing the buoyancy, the robot tends to float upward; however, the fins force the machine to move forward at the same time (similar in some sense to an airplane). After moving forward and vertically for a desired amount, the buoyancy can be altered again to force the robot to dive-again, the robot moves forward in conjunction with the descent. Combining these motions, the robot can traverse large distances across the ocean, simply by using repeated ascents and descents through controlled buoyancy. In addition, a controlled set of weights inside the body allows the center of gravity to be shifted to allow for either steeper or shallower ascents/descents and for turning. The entire device requires no externally actuated control or propulsion surfaces, and so is capable of very quiet operations.

\section{Aerial and Aerospace Robotic Locomotion}

"Flying. Whatever any other organism has been able to do man should surely be able to do also, though he may go a different way about it." - Samuel Butler

Modern robots can walk, crawl, roll, and swim, so why not fly? In fact, aerial and aerospace robotics is an area that is currently seeing a rapid expansion in research. The standard, or what we might loosely refer to as "conventional," modes of locomotion in this context are those most commonly used for aerospace transportation in the commercial sector; namely, airplanes and helicopters. The military has had obvious interest in autonomous flying drones for many years, with mixed results in implementation. The use of a helicopter as a platform for flying has also seen a burst of activity, originally inspired by the Autonomous Unmanned Vehicles (AUV) Aerial Robotics Competition started at the Georgia Institute of Technology, and currently being sponsored by the University of Florida. Since then, many aircraft of 
different sizes and shapes have been introduced to solve the problem of controlled autonomous flight.

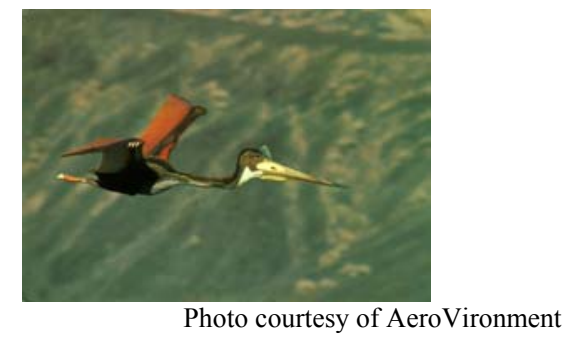

Figure 13: Pterodactyl robot in flight

The underlying desire to fly is one that is undeniable - it takes us to places we could not otherwise reach, and allows us to understand our world and other words in new and different ways. We focus here only on some of the forms of flight that use less conventional techniques for mobility. In what might be considered the "oldest" form of flight that has been explored, AeroVironment has developed a flying Pterodactyl, shown in Figure 13. Although primarily a glider, this creature was able to take wing and soar through the air using radio $(\mathrm{R} / \mathrm{C})$ controls.

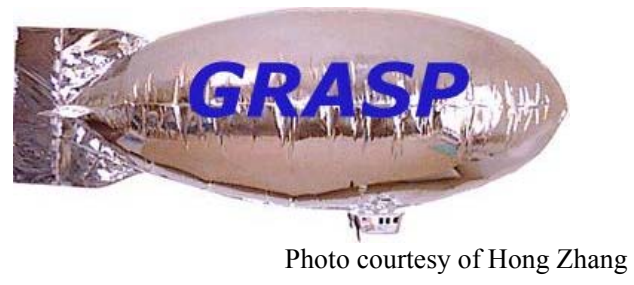

Figure 14: Vision-guided blimp robot

There are, of course, also much more modern examples of unconventional robotic systems capable of fully spatial motions. The use of airships, or blimps, for example, has seen a variety of uses, primarily because they can yield very high efficiency, a stable platform for observations, and can also be used in configurations near humans. Canny at
University of California at Berkeley, for example, has developed indoor blimps that can be remotely piloted over the internet, and thus provide a mechanism for telepresence, enabling a remote user to virtually participate in a physical setting. Ostrowski and Zhang, as well as a number of researchers in Brazil have also developed blimp-like vehicles (see Figure 14) capable of autonomous flight, tracking, and navigation, that can be used to perform mapping or even monitor critical areas, such as the tropical rainforests.
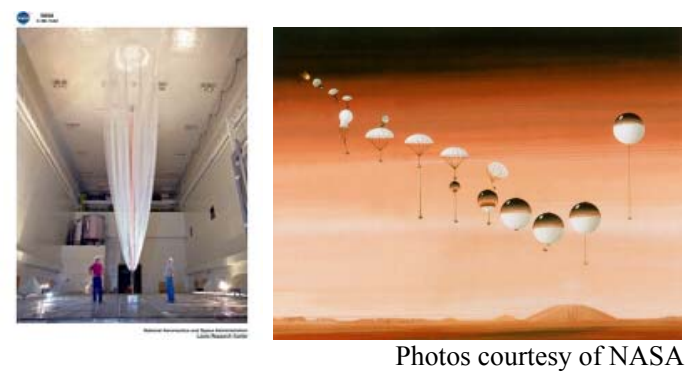

Figure 15: Mars Aerobot and illustration of initial deployment

Another very unusual blimp-like vehicle is the Aerobot, designed by NASA to do mapping and exploration here on Earth or on other planets. Shown in Figure 15 is a picture of a $10 \mathrm{~m}$ tall prototype, as well as an artist's rendition of how such a robot is deployed in the upper atmosphere. The primary concept is similar to the one used by Leonard in designing the swimming devices - changes in buoyancy are relatively cheap, and so vertical motion of the robot is easily and efficiently accomplished. The hard part tends to be how to move the robot laterally. For this, the Aerobot relies on existing air currents in the atmosphere, and jumps from stream to stream, just by changing altitude. The underlying concept was used in the Venus Vega 
mission, though the full control of the Aerobot's mobility will not be tested until the upcoming Mars missions scheduled for 2003.

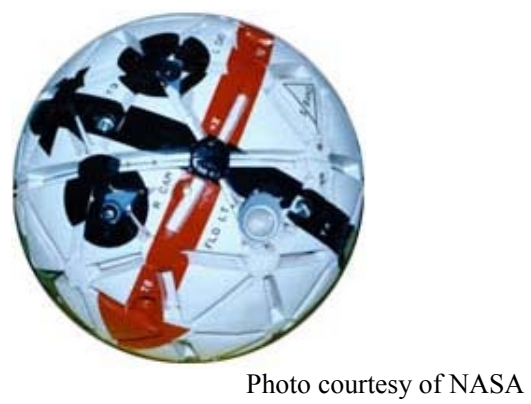

Figure 16: Sprint AERCam space robot

The outer reaches of space are also the domain of current day robots. Generally these robots fall into the terrestial category, since, like the highly successful Mars Rover, they are wheel-based. However, there are some robots that are venturing out in space as true flying agents. These are exemplified by the Sprint AerCAM, which is designed as a floating personal assistant for the astronauts working in space and on the International Space Station. Such robotic systems are close cousins to autonomously navigating satellitesrecent technology has focused on developing formations of microsatellites that can provide the same coverage and baseline separation as a much larger, single satellite.

\section{Hints of the Future}

"Telling the future by looking at the past assumes that conditions remain constant. This is like driving a car by looking in the rearview mirror."

\section{- Herb Brody}

What unique and surprising unconventional systems will the future bring? Looking in our rearview mirror, it is clear that the current trend in new robotic systems is centered on biomimetics - nature has evolved a wondrously diverse set of highly adapted mechanisms for locomotion, and we are only just now tapping into this powerful source of designs. As engineers, however, the next step is to improve on what we have observed. We are hardly at the stage where we can claim to have made many things better than those found in nature, but we are very close to the crossroads.

Some other examples of biomimetic systems not addressed above include two systems developed by Burdick and others at the California Institute of Technology. The hopping robot, shown in Figure 17, is capable of hopping across distances up to $2 \mathrm{~m}$, and is designed for use in exploring uneven terrain on missions to Mars or other planets. It also possesses a unique design that allows it to jump, right itself on the ground, scan the scene for interesting features, and then jump again, all using a single motor. This is an excellent example of minimalism in design and actuation, which is a current trend in robotic design.
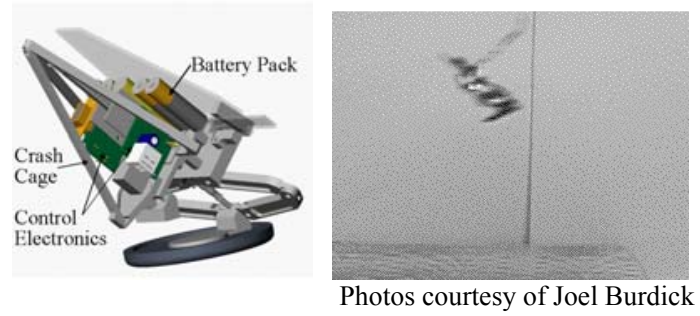

Figure 17: Hopping frog robot (right), and in flight (left)

Burdick also developed the inchworming device shown in Figure 18. This device belongs to a class of robotic systems that have been developed to locomote through closed surfaces such as pipes, tubes, or in this case, the small 
and large intestines. The inchworming robot can guide a small endoscope through the curvy path of the intestines, exploring areas that could not be reached with a traditional, unactuated endoscope. The locomotion for the inchworm is performed by expanding balloons to press against the walls of the intestines, combined with appropriate extensions of the individual segments, leading to an inchworming gait. Many other groups, including Foster-Miller, have developed robots for moving through pipes or for burrowing like an earthworm underground.

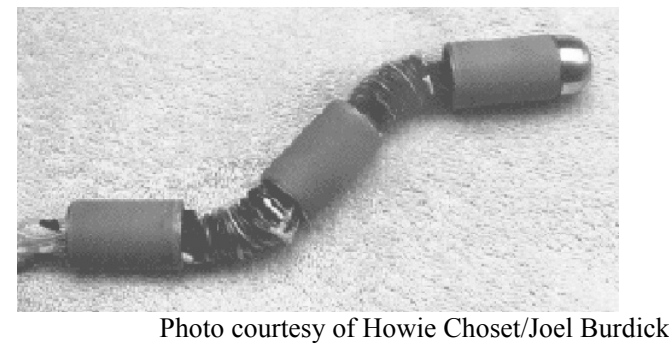

Figure 18: Intestinal inchworm robot

A second trend that is clear is the move towards miniaturization. The area of Micro-Electromechanical Systems (MEMS) is one that has pushed the size scales of our robotic systems into new domains. As robotics become smaller and smaller, we must now turn to microscopes to see many of the new creations. This will lead to robotic devices capable of swimming through our blood stream to deliver drugs or monitor blood chemistry, floating on air currents, and possibly fighting diseases or other micro-battles.

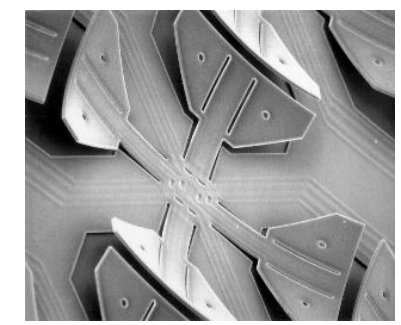

Figure courtesy of Bruce Randall Donald; reprinted, with permission, from "CMOS Integrated Organic Ciliary Array

for General-Purpose Micromanipulation Tool for Small

Objects," by J. Suh, R.B. Darling, K.-F. Bohringer, B. R. Donald, H. Baltes, and G. Kovacs, Journal of Microelectomechanical Systems, Vol. 8, No. 4 (Dec. 1999), pp. 483 - 496, http://www.cs.dartmouth.edu/ brd/Research/ MEMS/ciliaarrays.html

Figure 19: Finger-like cilia for manipulation and, potentially, mobility

We are still a good deal away from this, but work on microswimmers by Fukuda at Nagoya University; fingerlike arrays and scratch actuators by Donald, Bohringer, and others at Dartmouth; and many others are the first steps toward making these devices a reality. For example, finger-like cilial arrays such as the ones developed by Bohringer and Donald shown in Figure 19 , are already capable of moving small parts across their surface. It is not a large stretch to imagine such devices flipped over to become cilial-based walking or inchworming mechanisms. There is also an extensive theory developed to describe locomotion at such scales, leaving an open question as to how this will be put into practice.

Microsystems are also the focus of many efforts to achieve new forms of flight. Some of these are referred to as "micro" systems because the scale is dramatically smaller than the any existing technology. They are perhaps more appropriately referred to as "mini" systems, though, to distinguish them from devices that require microscopes to properly be viewed. An example of one such "mini" robotic system is AeroVironment's Yellow Jacket (shown 
in Figure 20) micro aerial vehicle (MAV's), which is capable of over 20 minutes of sustained flight, carrying an onboard camera. Another type of "mini" robotic system is the new wave of microsatellites (generally "micro" means payloads of about $1 \mathrm{~kg}$ or less) being developed for use in communications and astronomical observations.

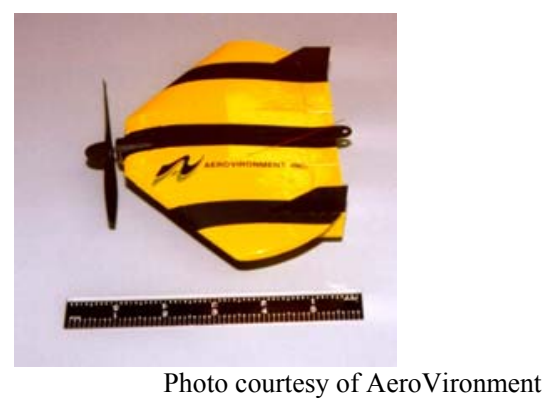

Figure 20: The Yellow Jacket MAV. This delta wing shaped aircraft features an advanced auto-stability system using a miniaturized rate gyro.

There are also a number of researchers who have begun to investigate biomimetic microflight, with systems ranging from small bats to the work at Berkeley by Fearing, Pister, and others on MEMS-based insect flight. While these systems are generally only in the experimental stages, it is clear that it will not be long before they are able to drop their tethers and reach for the sky.

\section{REFERENCES}

Alexander, R.M. (1984). The gaits of bipedal and quadrupedal animals, International Journal of Robotics Research, 3(2), pp. 49-59.
Chirikjian, G.S. (2000) Design and Analysis of Some Nonanthropomorphic BiologicallyInspired Robots. To appear in the Journal of Intelligent and Robotic Systems (special issue on Anthropomorphic Robots, Jadran Lenarcic guest editor).

Collins, J.J. and Stewart, I. (1993) Coupled Nonlinear Oscillators and the Symmetries of Animal Gaits. Journal of Nonlinear Science, 3(3), pp. 349-392.

Hirose, S. (1993). Biologically inspired robots: Snake-like locomotors and manipulators. Translated by Peter Cave and Charles Goulden. Oxford University Press, Oxford; New York.

Marsden, J.E. and Ostrowski, J. (1998). Symmetries in motion: Geometric foundations of motion control. Nonlinear Science Today. Available electronically at http://www.springer-ny.com/nst.

Ostrowski, J. P. and Burdick, J. W. (1998). The geometric mechanics of undulatory robotic locomotion. International Journal of Robotics Research 17(7), pp. 683-701.

Song, S.-M. and Waldron, K.J. (1989) Machines that walk: The adaptive suspension vehicle. MIT Press, Cambridge, MA. 